American J. of Engineering and Applied Sciences 5 (1): 42-48, 2012

ISSN 1941-7020

(C) 2014 J.X. Rodrigues et al., This open access article is distributed under a Creative Commons Attribution

(CC-BY) 3.0 license

\title{
Sharp Transition Multiband Filter in Speech Processing Scheme for Hearing Impaired
}

\author{
${ }^{1}$ Joseph X. Rodrigues, ${ }^{2}$ Lucy J. Gudino and ${ }^{3}$ K.R. Pai \\ ${ }^{1}$ Department of Electronics and Communications, \\ Agnel Technical Education Complex, Verna, Goa, India \\ ${ }^{2}$ Department of Computer Science and Information Sciences, \\ BITS Pilani-K.K. Birla Goa Campus, Goa, India \\ ${ }^{3}$ Department of Electronics and Telecommunications Enginnering, \\ P.C. College of Engineering, Verna, Goa, India
}

\begin{abstract}
Problem statement: A simple analytical approach to the synthesis of a sharp transition, linear phase, multiband FIR filter is presented. The filter magnitude response is modeled using trigonometric functions of frequency. Approach: Employing variable density of ripple cycles in passband and stopband regions with large density of ripples cycles at the sharp transition edges, reduces the abrupt discontinuities at these edges. Results: As a result, Gibb's phenomenon is reduced in the filter implementation thus giving a flat passband and good stopband attenuation. A closed form expression for impulse response coefficients is obtained. The filter design is easily tunable and allows for variation in transition bandwidth of each band. A speech processing scheme is implemented using a pair of the proposed sharp transition multiband FIR filters to split the speech spectrum into complementary short time spectral bands. Conclusion: The adjacent speech formants are fed dichotically to the two ears to reduce the effect of spectral masking and hence improve speech perception in sensorineural hearing impaired.
\end{abstract}

Key words: Sharp transition, FIR filter, multiband filter, gibb's phenomenon, sensorineural hearing impaired, speech processing scheme

\section{INTRODUCTION}

The peripheral auditory system of the human ear behaves like a bank of bandpass filters called auditory filters or critical bands (Moore, 2003; Flanagan, 1972; Zwicker, 1961).One of the hearing impairment in humans is sensorineural hearing loss due to defects in the cochlea and auditory nerve. In general, this loss is not medically curable and it becomes progressively worse with time. Sensorineural loss is associated with widening of the auditory filter bandwidths thus the filter slope becomes shallower and results in overlap of adjacent spectral bands called spectral masking (Boer and Bouwmeester, 1974; Glasberg and Moore, 1986; French and Steinberg, 1947). This leads to a decrease in frequency resolving capacity of the auditory system of the ears. One of the possible ways of improving speech perception would be to split the speech signal on the basis of its short time spectrum into two complimentary spectra for presentation to the two ears dichotically. This enhances the perception of spectral contrast of resonance peaks without adversely affecting the perception of features cued by amplitude and duration. The two adjacent strong spectral components i.e. formants that are likely to mask each other are presented to left and right ears. Another technique to reduce effects of spectral masking is to employ multiband frequency compression (Kulkarni et al., 2007). In this technique, the speech spectrum is divided into a number of analysis bands and spectral samples in each of these bands are compressed towards the band center by a constant compression factor.

Several schemes are employed in literature to split the speech signals for binaural dichotic presentation. Lunner et al. (1993) evaluated a scheme using synthesized vowels in which the first formant is presented to left ear and the second formant to the right ear. The scheme used comb filters with constant bandwidth of $700 \mathrm{~Hz}$ and $40 \mathrm{~dB}$ stopband attenuation. The listening tests were conducted on male and female subjects in age group of 39-69 years with bilateral and moderate sensorineural hearing loss. The gain of the filters was adjusted depending on the hearing loss of

Corresponding Author: Joseph X. Rodrigues, Department of Electronics and Communications,

Agnel Technical Education Complex, Verna, Goa, India 
individual subject. The scheme achieved spectral splitting but the filters did not have the sharp transition bands. The results indicated an overall improvement in speech perception for dichotic conditions. Lyregaard (1982) conduced similar experiments with three values of comb filter bandwidths of $200 \mathrm{~Hz}, 500 \mathrm{~Hz}$ and $800 \mathrm{~Hz}$. There was no significant improvement in speech recognition scores because of improper band separation. Chaudari and Pandey (1998) investigated a scheme for splitting the speech into two complementary spectra each with nine bands for binaural dichotic presentation using Zwicker (1961) psychophysical tuning curves. The FIR comb filters were designed by frequency sampling method using linear optimization techniques. The filter transition bandwidth was 78 to $117 \mathrm{~Hz}$ for various bands which resulted in maximum pass-band ripple of $1 \mathrm{~dB}$ and stopband attenuation of 38 $\mathrm{dB}$ with cascade filters of order 256 . The scheme was able to improve speech quality, response time, recognition scores and transmission of consonantal features as observed in the listening tests. The criticalband filter banks for use in binaural hearing aid reported in literature generally uses lowpass, bandpass and highpass filter in cascade. These filters have a wide transition band, large passband ripple, less stopband attenuation and not easily tunable. Employing filters with large transition band, the spectral components lying in the transition region are presented to both ears which impair speech intelligibility (Chaudari and Pandey, 1998).

A multiband sharp transition filter with large number of passbands and acceptable magnitude response is difficult to design and requires large filter order (Pelloni and Lagadec, 1984). Most methods do not allow independent control of band specifications mainly the transition bandwidth. Conventional methods for sharp transition FIR filters using window functions and frequency sampling method require high filter order. The numeric Remez approach, which employs optimization techniques, requires lower filter order but computational complexity is high. They do not yield an explicit expression for the filter transfer function and hence it is difficult tuning to tailor made magnitude responses desired in some applications like the one dealt in this study. Most of the numeric FIR multiband filter approaches (Rabiner et al., 1974; Zahradnik and Vleek, 2005; Mintzer and Liu, 1979; Matei, 2007; Shpak and Antoniou, 1988; Selesnick et al., 1998; Burrus, 1994; Estola, 1988; Samadi et al., 2004; Ho et al., 2008) are based on the least squares or equiripple error criteria. A Remez type exchange algorithm with an initialization strategy and a selective search for the location of the error maxima magnitude to reduce the computation complexity is presented by Shpak and Antoniou (1988). A simple and effective multipleexchange algorithm for the design of multiband FIR filter is presented by Selesnick et al. (1998) using a constrained least square approach that does not use specified transition bands. The design algorithm is a hybrid of the Parks-McClellan and least squares methods. Two methods which are a combination of numeric and analytical techniques are presented by Burrus (1994) for designing optimal linear phase multiband FIR digital filters that have well defined transition bands. They both allow explicit control of band edges and center band gains for multiple passband and independent control of each transition band edge. One is an analytical method which derives formulas for the filter coefficients but does not allow different weighting of the passbands, stopbands or transition bands. The second approach uses a mixed numericalanalytical method which allows independent weighting of all bands but requires numerical solution of a set of simultaneous equations. A general method is presented by Estola (1988) for designing computationally efficient FIR multiband filters with arbitrary frequency response and time response. These filters are constructed by cascading subfilters and solving the nonlinear approximation problem to obtain filter coefficients. A linear phase maximally flat multiband filter is presented by Samadi et al. (2004). The structure is easily tunable and provides a simple mechanism to vary both the centers and degrees of flatness independently at each level. A serious disadvantage of FIR filters is its complexity, which becomes acute in sharp trasition filters. A computationally efficient design technique for synthesis of sharp transition FIR filters is Interpolated FIR filter which is a FIR-FIR filter cascade and employs sparse filter and an interpolator filter (Neuvo et al., 1987; 1984; Jovanovic-Dolecek and Diaz-Carmona, 2002; Yang and Lian, 2002; Gudino et al., 2008a; 2008b; 2008c). The interpolation designs introduce large group delays. One of the most successful techniques for the synthesis of arbitrary passband, very narrow transition width FIR filter with least filter order is the Frequency Response Masking (FRM) technique (Lim, 1986; Rodrigues and Pai, 2005a; Lim et al., 2007). The sharp transition and arbitrary bandwidth is formed by using several low order FIR subfilters. Hearing aids using a bank of computationally efficient FRM filters but with wider transition band are presented by Lian and Wei (2005). 


\section{MATERIALS AND METHODS}

Proposed multiband filter design: In the proposed linear phase FIR multiband filter design, the various regions of the filter magnitude response are modeled using trigonometric functions of frequency. The magnitude response model $H_{p m}(\omega)$ of the multiband filter shown in Fig. 1 has nine regions per band. $\mathrm{BP}_{\mathrm{n}}$ is the nth bandpass component of the multiband filter.

In the stopband region from $\omega_{\mathrm{s}, \mathrm{n}-1} \leq \omega \leq \omega_{\mathrm{s} 2, \mathrm{n}-1}$ the frequency response is Eq. 1:

$$
\mathrm{H}_{\mathrm{pm}}\left(\omega=\frac{\delta_{\mathrm{s}}}{2}\right) \cos \mathrm{k}_{\mathrm{p} 2}\left(\omega-\omega_{\mathrm{s}, \mathrm{n}-1}\right)
$$

where, $\omega$ is the frequency variable, $\delta_{\mathrm{s}}$ is the stopband attenuation, $\omega_{\mathrm{s}, \mathrm{n}-1}$ is the center frequency of the stop band preceding a passband of bandpass response $\mathrm{BP}_{n}$ and $\omega_{\mathrm{s} 2 \mathrm{n}-1}$ is the frequency at which second stopband region starts. This region is designed to possess (n0-p) ripple cycles characterized by filter design parameter $\mathrm{k}_{\mathrm{p} 2}$ where $\mathrm{n} 0$ and $\mathrm{p}$ are integers to be chosen. The various filter design parameters of the passband, stopband and transition bands shape the response in the respective regions Eq.2:

$\omega_{\mathrm{s} 2, \mathrm{n}-1}=\omega_{\mathrm{s}, \mathrm{n}-1}+(\mathrm{n} 0-\mathrm{p}) \frac{2 \pi}{\mathrm{k}_{\mathrm{p} 2}}$

For the filter model, $\mathrm{H}_{\mathrm{pm}}\left(\omega_{\mathrm{bn}} \pm \omega_{\mathrm{pn}}\right)=1$ and $\mathrm{H}_{\mathrm{pm}}\left(\omega_{\mathrm{bn}} \pm \omega_{\mathrm{zn}}\right)=0$.

In the stopband region, $\omega_{\mathrm{s} 2, \mathrm{n}-1} \leq \omega \leq\left(\omega_{\mathrm{bn}}-\omega_{\mathrm{zn}}\right)$ the frequency response is Eq. 3:

$\mathrm{H}_{\mathrm{pm}}(\omega)=\frac{\delta_{\mathrm{s}}}{2} \cos \mathrm{k}_{\mathrm{p}}\left(\omega-\omega_{\mathrm{s} 2, \mathrm{n}-1}\right)$

where, $\omega b_{n}$ is the center frequency of passband $\mathrm{BP}_{\mathrm{n}}$. This region possesses $(\mathrm{p}+3 / 4)$ cycles of ripple characterized by filter design parameter $\mathrm{kp}$.

For the filter model Eq. 4 and 5:

$$
\begin{aligned}
& \left(\omega_{\mathrm{bn}}-\omega_{\mathrm{zn}}\right)-\omega_{\mathrm{s}, \mathrm{n}-1}=\left(\mathrm{p}+\frac{3}{4}\right) \frac{2 \pi}{\mathrm{k}_{\mathrm{p}}}+(\mathrm{n} 0-\mathrm{p}) \frac{2 \pi}{\mathrm{k}_{\mathrm{p} 2}} \\
& \omega_{\mathrm{s} 2, \mathrm{n}-1}=\left(\omega_{\mathrm{bn}}-\omega_{\mathrm{zn}}\right)-\left(\mathrm{p}+\frac{3}{4}\right) \frac{2 \pi}{\mathrm{k}_{\mathrm{p}}}
\end{aligned}
$$

In the transition region, $\left(\omega_{\mathrm{bn}}-\omega_{\mathrm{zn}}\right) \leq \omega \leq\left(\omega_{\mathrm{bn}}-\omega_{\mathrm{pn}}\right)$, the frequency response is Eq. 6:

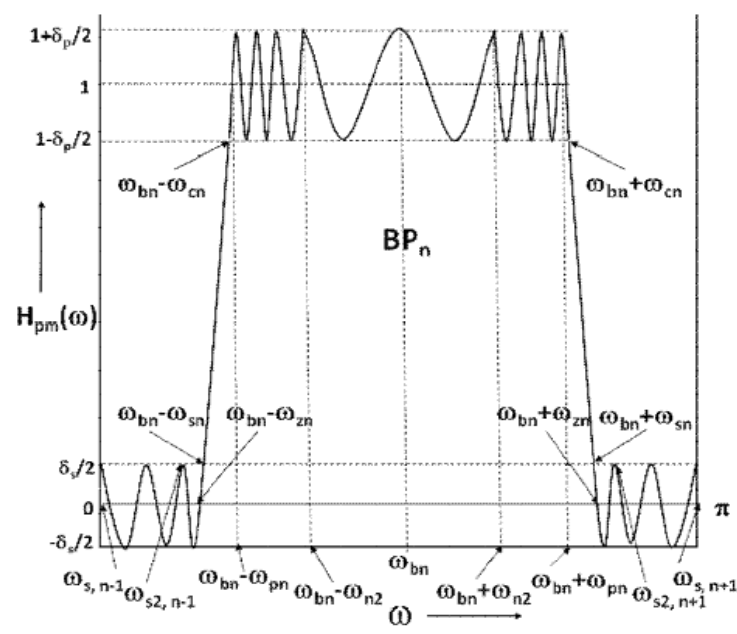

Fig. 1: Illustration of one band of the proposed ' $n$ ' band multiband FIR filter with equiripple magnitude response, linear transition and variable density ripple cycles

$\mathrm{H}_{\mathrm{pm}}(\omega)=\frac{1}{\left(\omega_{\mathrm{zn}}-\omega_{\mathrm{pn}}\right)}\left[\omega-\left(\omega_{\mathrm{bn}}-\omega_{\mathrm{zn}}\right)\right]$

where, $\omega_{\mathrm{bn}} \pm \omega$ p are the passband edge frequencies and $\omega_{\mathrm{pn}}$ is half passband width of $\mathrm{BP}_{\mathrm{n}}$.

In the passband region, $\left(\omega_{\mathrm{bn}}-\omega_{\mathrm{pn}}\right) \leq \omega \leq\left(\omega_{\mathrm{bn}}-\omega_{\mathrm{n} 2}\right)$, the frequency response is Eq. 7:

$\mathrm{H}_{\mathrm{pm}}(\omega)=1+\frac{\delta_{\mathrm{p}}}{2} \sin \mathrm{k}_{\mathrm{p}}\left[\omega-\left(\omega_{\mathrm{bn}}-\omega_{\mathrm{pn}}\right)\right]$

where, $\left(\omega_{\mathrm{bn}}-\omega_{\mathrm{n} 2}\right)$ is the frequency at which second passband region starts. This region possesses $(m+1 / 4)$ cycles of ripple and $\mathrm{m}$ is an integer to be chosen.

In the passband region, $\left(\omega_{\mathrm{bn}}-\omega_{\mathrm{n} 2}\right) \leq \omega \leq\left(\omega_{\mathrm{bn}}-\omega_{\mathrm{n} 2}\right)$, the frequency response is Eq. 8:

$\mathrm{H}_{\mathrm{pm}}(\omega)=1+\frac{\delta_{\mathrm{p}}}{2} \operatorname{cosk}_{\mathrm{p} 1}\left[\omega-\left(\omega_{\mathrm{bn}}-\omega_{\mathrm{n} 2}\right)\right]$

where, $\left(\omega_{\mathrm{bn}}+\omega_{\mathrm{n} 2}\right)$ is the frequency at which third passband region starts. This region possesses $(\mathrm{n} 1-2 \mathrm{~m})$ cycles of ripples and is characterized by the filter design parameter kp1. $\mathrm{n} 1$ is an even integer to be chosen.

For the filter model Eq. 9:

$\omega_{\mathrm{n} 2}=\left(\frac{\mathrm{n} 1}{2}-\mathrm{m}\right) \frac{2 \pi}{\mathrm{k}_{\mathrm{p} 1}}$

In the passband region, $\left(\omega_{\mathrm{bn}}+\omega_{\mathrm{n} 2}\right) \leq \omega \leq\left(\omega_{\mathrm{bn}}+\omega_{\mathrm{pn}}\right)$, the frequency response is Eq. 10: 
$\mathrm{H}_{\mathrm{pm}}(\omega)=1+\frac{\delta_{\mathrm{p}}}{2} \cos \mathrm{k}_{\mathrm{p}}\left[\omega-\left(\omega_{\mathrm{bn}}+\omega_{\mathrm{n} 2}\right)\right]$

This region is designed to possess $(m+1 / 4)$ cycles of ripple Eq. 11:

$\omega_{\mathrm{n} 2}=\omega_{\mathrm{pn}}-\left(\mathrm{m}+\frac{1}{4}\right) \frac{2 \pi}{\mathrm{k}_{\mathrm{p}}}$

In the transition region, $\left(\omega_{\mathrm{bn}}+\omega_{\mathrm{pn}}\right) \leq \omega \leq\left(\omega_{\mathrm{bn}}+\omega_{\mathrm{zn}}\right)$, the frequency response is Eq. 12:

$\mathrm{H}_{\mathrm{pm}}(\omega)=1-\frac{1}{\left(\omega_{\mathrm{zn}}-\omega_{\mathrm{pn}}\right)}\left[\omega-\left(\omega_{\mathrm{bn}}+\omega_{\mathrm{pn}}\right)\right]$

In the stopband region, $\left(\omega_{\mathrm{bn}}+\omega_{\mathrm{zn}}\right) \leq \omega \leq \omega_{\mathrm{s} 2, \mathrm{n}-1}$, the frequency response is Eq. 13:

$\mathrm{H}_{\mathrm{pm}}(\omega)=-\frac{\delta_{\mathrm{s}}}{2} \sin \mathrm{k}_{\mathrm{p}}\left[\omega-\left(\omega_{\mathrm{bn}}+\omega_{\mathrm{zn}}\right)\right]$

where, $\omega_{\mathrm{s} 2, \mathrm{n}-1}$ the frequency at which third region of the stopband starts. This region is designed to possess $(\mathrm{q}+3 / 4)$ cycles of ripple characterized by $\mathrm{k}_{\mathrm{p}}$ and $\mathrm{q}$ is an integer is to be chosen.

In the stopband region, $\omega_{\mathrm{s} 2, \mathrm{n}-1} \leq \omega \leq \omega_{\mathrm{s}, \mathrm{n}-1}, \quad$ the frequency response is Eq. 14:

$\mathrm{H}_{\mathrm{pm}}(\omega)=\frac{\delta_{\mathrm{s}}}{2} \sin \mathrm{k}_{\mathrm{p} 3}\left(\omega-\omega_{\mathrm{s} 2, \mathrm{n}+1}\right)$

$\omega_{\mathrm{s}, \mathrm{n}-1}$ is the center frequency of the stopband succeeding the band BPn. This region is designed to possess (n2-q) cycles of ripple characterized by filter design parameter $\mathrm{k}_{\mathrm{p} 3}$ and $\mathrm{n} 2$ is an integer to be chosen. For the filter model Eq.15:

$$
\begin{gathered}
\omega_{\mathrm{s}, \mathrm{n}-1}-\left(\omega_{\mathrm{bn}}+\omega_{\mathrm{zn}}\right)=\left(\mathrm{q} \frac{3}{4}\right) \frac{2 \pi}{\mathrm{k}_{\mathrm{p}}} \\
\left(\mathrm{n}_{2}-\mathrm{q}\right) \frac{2 \pi}{\mathrm{k}_{\mathrm{p} 3}}
\end{gathered}
$$

For the filter model Eq. 16-18:

$$
\begin{aligned}
& \omega_{\mathrm{s}, \mathrm{n}-1}-\left(\omega_{\mathrm{bn}}+\omega_{\mathrm{zn}}\right)=\left(\mathrm{q} \frac{3}{4}\right) \frac{2 \pi}{\mathrm{k}_{\mathrm{p}}} \\
& \omega_{\mathrm{cn}}=\frac{\delta_{\mathrm{p}}}{2}\left(\omega_{\mathrm{zn}}-\omega_{\mathrm{pn}}\right)+\omega_{\mathrm{pn}} \\
& \omega_{\mathrm{sn}}-\omega_{\mathrm{zn}}-\left(\delta_{\mathrm{s}} / 2\right)\left(\omega_{\mathrm{zn}}-\omega_{\mathrm{pn}}\right)
\end{aligned}
$$

The transition bandwidth of each band is Eq. 19: $\left(\omega_{\mathrm{sn}}-\omega_{\mathrm{cn}}\right)=\left(\omega_{\mathrm{zn}}-\omega_{\mathrm{pn}}\right)\left(1-\frac{\delta_{2}+\delta_{\mathrm{p}}}{2}\right)$

For the multiband design, novel slope equalization technique (Rodrigues and Pai, 2005b) is applied which further improves the performance of the filter. The filter design parameter $\mathrm{k}_{\mathrm{p}}$ is evaluated by equalizing the slopes of the magnitude response function at the edges of the transition region which are the points of discontinuity. This allows the function to be continuous thus reducing the effects due to Gibb's phenomenon and hence improves performance of the filter Eq. 20 and 21:

Slope of $H_{p m}(\omega)$ at $\omega_{b n} \pm \omega_{p n}=-\frac{k_{p} \delta_{p}}{2}$

Slope of $H_{\mathrm{pm}}(\omega)$ at $\omega_{\mathrm{bn}} \pm \omega_{\mathrm{zn}}=-\left(-1 /\left.\omega\right|_{\mathrm{zn}}-\omega_{\mathrm{pn}}\right)$

Equating the slopes at $\left(\omega_{\mathrm{bn}}+\omega_{\mathrm{pn}}\right)$ and $\left(\omega_{\mathrm{bn}}+\omega_{\mathrm{zn}}\right)$ at the points of discontinuity for each band of the multiband filter and using (20),(21) and (19) we obtain Eq. 22:

$\mathrm{k}_{\mathrm{p}}=\frac{2\left[1-\left(\frac{\delta_{\mathrm{s}}+\delta_{\mathrm{p}}}{2}\right)\right]}{\delta_{\mathrm{p}}\left(\omega_{\mathrm{sn}}-\omega_{\mathrm{cn}}\right)}$

This value of $\mathrm{kp}$ further reduces ripples at the transition edges hence reduces passband ripple and improves stopband attenuation.

The expression for the impulse response coefficients for the $\mathrm{N}$ order multiband filter is obtained by evaluating the integral Eq. 23:

$\mathrm{h}\left(\mathrm{n}_{\mathrm{i}}\right)=\frac{1}{\pi}\left[\int_{0}^{\pi} \mathrm{H}_{\mathrm{pm}}(\omega) \cos \mathrm{k} \omega \mathrm{d} \omega\right]$

where, $\mathrm{H}_{\mathrm{pm}}(\omega)$ in 23 is made up of nine regions for each passband as defined by $1,3,6-8,10,12-14$. $\mathrm{n}_{\mathrm{i}}=0,1, \ldots ., \frac{\mathrm{N}-1}{2}$ for $\mathrm{N}$ odd and $\mathrm{k}=\frac{(\mathrm{N}-1)}{2}-\mathrm{n}_{\mathrm{i}}$.

Using Eq. 23, the symmetric impulse response coefficients for a given filter order $\mathrm{N}$ can be obtained.

\section{RESULTS}

Design two linear phase, sharp transition FIR multiband filters using the proposed design approach to be employed in a speech processing scheme for sensorineural hearing impaired. The speech samples are 
split into two complementary short-time spectra using the multiband FIR filters with passbands based on Zwicker's critical band model (Zwicker, 1961). Each multiband filter has nine passbands with passband edge frequencies. . $\omega_{\mathrm{bn}} \pm \omega_{\mathrm{pn}}$ in $\mathrm{KHz}$ being [0.07-0.2, 0.30$0.40,0.51-0.63,0.77-0.92,1.08-1.27,1.48-1.72,2.00-$ $2.32,2.70-3.15,3.70-4.40]$ and $[0.2-0.3,0.40-0.51$, $0.63-0.77,0.92-1.08,1.27-1.48,1.72-2.00,2.32-2.70$, $3.15-3.70,4.40-5.012]$, sampling frequency of 11025 $\mathrm{Hz}$, transition bandwidth. . $\omega_{\mathrm{sn}}-\omega_{\mathrm{cn}}$ of $35 \mathrm{H}_{\mathrm{z}}$, maximum passband ripple $\delta_{\mathrm{p}}$ is $\pm 0.1 \mathrm{~dB}$ and minimum stopband attenuation $\delta_{\mathrm{s}}$ is $40 \mathrm{~dB}$.

The specification of each band i.e., passband center frequency $\omega_{\mathrm{bn}}$, half passband width $\omega_{\mathrm{pn}}$, the center frequencies of the stopbands preceding and succeeding $\mathrm{B}_{\mathrm{Pn}} \cdot \omega_{\mathrm{s}, \mathrm{n}-1}$ and $\omega_{\mathrm{s}, \mathrm{n}-1}$ can be found from the passband values given above. For the given filter specifications, we compute values of $\mathrm{k}_{\mathrm{p}}, \omega_{\mathrm{zn}}, \omega_{\mathrm{n} 2}, \mathrm{k}_{\mathrm{p} 1}, \omega_{\mathrm{s} 2, \mathrm{n}-1}, \mathrm{k}_{\mathrm{p} 2}, \omega_{\mathrm{s} 2, \mathrm{n}-1}$ and $\mathrm{k}_{\mathrm{p} 3}$ using Eqs. 22, 19, 11, 9, 5, 2, 16 and 15 respectively. The value of the chosen constants $m, n 1$, $\mathrm{p}, \mathrm{q}, \mathrm{n} 0$ and $\mathrm{n} 2$ depends on the density of the ripples required in the various passbands and stopbands of the multiband filter. In the model, where sharp edges join, like the transition and first passband region, the ripples are increased. Hence a higher value of the constant is chosen in this region. The number of constants depends on the number of passbands. The approximate relation between various constants is $\mathrm{n} 1>2 * \mathrm{~m}, \mathrm{p}=\mathrm{q}, \mathrm{n} 0>\mathrm{p}, \mathrm{q}$, $\mathrm{n} 2>\mathrm{p}, \mathrm{q}$. We choose $\mathrm{m}=30, \mathrm{n} 1=70, \mathrm{p}=\mathrm{q}=5, \mathrm{n} 0=7$ and $\mathrm{n} 2=15$.The magnitude response of the multiband filter obtained is shown in Fig. 2. The peak ripple at the transition edges due to Gibb's phenomenon reduces. The proposed design yields the desired multiband FIR filter specifications for a filter order of 1025 . The individual bands have a passband ripple of 0.01 $\mathrm{dB}$, stopband attenuation exceeding $40 \mathrm{~dB}$ and a sharp transition band of $35 \mathrm{~Hz}$ i.e., normalized frequency of 0.00635. In a similar speech processing scheme, (Kulkarni and Pandey, 2008) employs two comb FIR filters with transition bandwidth of $78-117 \mathrm{~Hz}$, passband ripple $<1 \mathrm{~dB}$ and stopband attenuation exceeding $30 \mathrm{~dB}$.

Listening tests were carried out on five subjects with bilateral moderate sensorineural hearing impairment. The test stimuli consisted of nonsense syllables formed with English consonants and vowel in vowel consonant vowel and consonant vowels contexts. Signal acquisition, administration of speech syllables and analysis was performed offline. Subjects showed an improvement in recognition of the speech syllables presented to the ears.

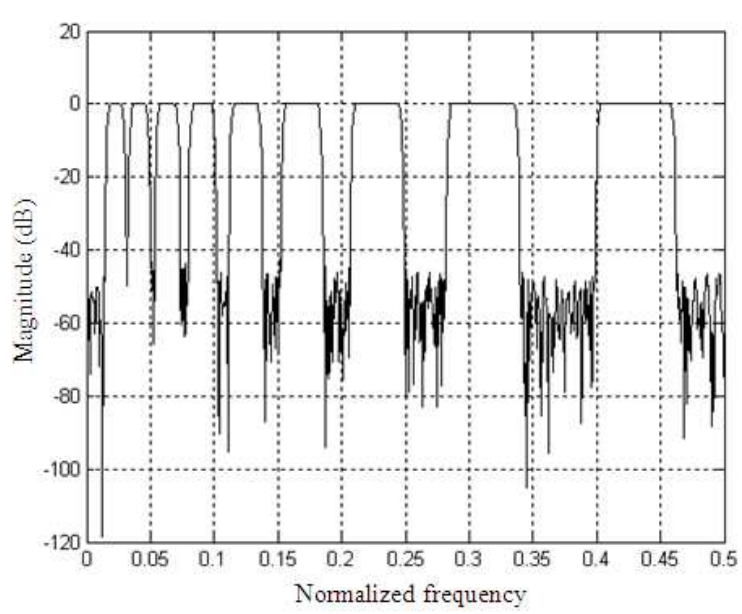

(a)

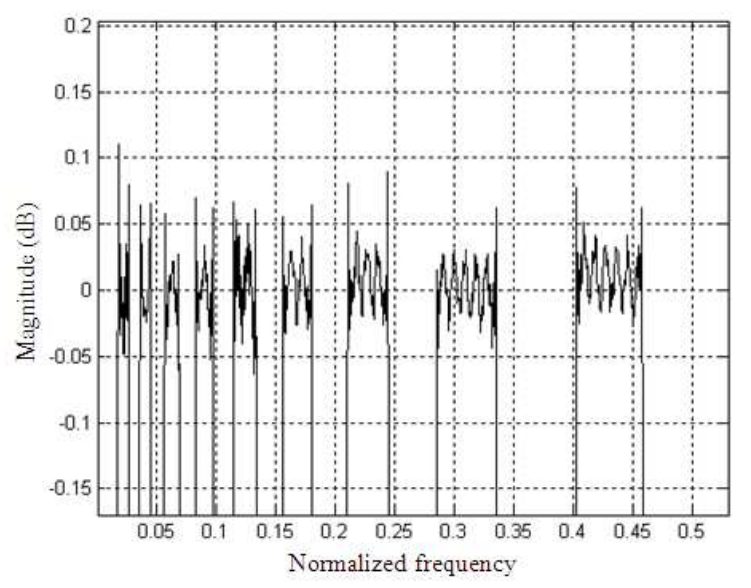

(b)

Fig. 2: (a) Magnitude response of the proposed FIR multiband filters for the design example (b) Magnified view of passband

For all the subjects the recognition scores for processed speech were higher than those for unprocessed speech. Response time to respond to speech syllables presented to the ears reduced which indicates effective speech processing. The processed speech was spectrographically analyzed to verify splitting of speech into various frequency bands. It was found that the adjacent formant frequencies of the speech syllables were presented to alternate ears thus aided in better recognition of speech using the proposed filters in the speech processing scheme.

\section{DISCUSSION}

FRM approach for a design of multiband filter with large number of passbands is complicated since many subfilters are to be designed. Also large delays 
introduced are undesired in real time applications. Synthesis of sharp transition FIR lowpass filters is introduced by Rodrigues and Pai, (2005a), Rodrigues et al. (2006). The simple analytical methods give narrow transition band with flat passband, good stopband attenuation with less filter order. The filter designs can be extended to the design of bandpass and multiband FIR filters. In this study, we propose the synthesis of sharp transition multiband FIR filter. The design permits easy tuning of the center frequency and transition bandwidths of individual bands.

\section{CONCLUSION}

This study presents the synthesis of a sharp transition, linear phase, multiband FIR filter. The filter is easily tunable, the ripples at the transition edges due to sharp transition are reduced, the design does not employ optimization techniques and we can implement tailor made filter magnitude responses. The specifications of each band i.e. center frequency, passband width, transition width and stopband attenuation can be easily varied. Proposed multiband filters have good stopband attenuation which aids in proper band separation and low passband ripple avoids deterioration of processed speech. Employing sharp transition filters aids in proper separation of adjacent formant frequencies fed to the ears. This leads to improvement in recognition of speech for sensorineural hearing impaired subjects as observed in the experimental study conducted.

\section{REFERENCES}

Burrus, C.S., 1994. Optimal least squares fir multiband filter design. Proceedings of the 6th IEEE Digital Signal Processing Workshop, Oct. 2-5, IEEE Xplore Press, Yosemite National Park, USA, pp: 215-218. DOI: 10.1109/DSP.1994.379838

Chaudari, D.S. and P.C. Pandey, 1998. Dichotic presentation of speech signal with critical band filtering for improving speech perception. Proceedings of International Conference on Acoustic, Speech and Signal Processing, May 1215, IEEE Xplore Press, Seattle, USA, pp: 36013604. DOI: 10.1109/ICASSP.1998.679658

Estola, K.P., 1988. Design of computationally efficient FIR filters for sampling rate alteration and multiband filtering with arbitrary passbands and time response. Proceedings of the IEEE International Conference on Acoustics, Speech and Signal Processing ICASSP, Apr. 1986, IEEE Xplore Press, pp: 2575-2578. DOI: 10.1109/ICASSP.1986.1169292

Flanagan, J.L., 1972. Speech Analysis Synthesis and Perception. 2nd Edn., Springer-Verlag, New York. ISBN-10: 0387055614 pp: 444.
French, N.R. and J.C. Steinberg, 1947. Factors governing the intelligibility of speech sounds. J. Acoust. Soc. Am., 19: 90-119. DOI: 10.1121/1.1916407

Glasberg, B.R. and B.C.J. Moore, 1986. Auditory filter shapes in subjects with unilateral and bilateral cochlear impairments. J. Acoust. Soc. Am., 79: 1020-1033. DOI: 10.1121/1.393374

Gudino, L.J., S.N. Jagadeesha and J.X. Rodrigues, 2008a. A new filter design for uniform linear array. IEEE International Conference on Systems, Signals and Devices IEEE SSD, July 20-22, IEEE Xplore Press, Amman, pp: 1-3. DOI: 10.1109/SSD.2008.4632883

Gudino, L.J., S.N. Jagadeesha and J.X. Rodrigues, 2008b. A novel design of sharp transition FIR filter for digital beamforming. 6th International Symposium on Communication Systems, Networks and Digital Signal Processing, July 25-25, IEEE Xplore Press, Graz, pp: 247-250. DOI: 10.1109/CSNDSP.2008.4610737

Gudino, L.J., S.N. Jagadeesha and J.X. Rodrigues, 2008c. A novel filter design for spatially interpolated beamformer. Proceedings of the International Symposium on Intelligent Signal Processing and Communication Systems, ISPACS, Feb. 8-11, IEEE Xplore Press, Bangkok, pp: 1-4. DOI: $10.1109 /$ ISPACS.2009.4806682

Ho, C.Y.F., B.W.K. Ling, Z.W. Chi, M. Shik-Bahaei and Y.Q. Liu et al., 2008. Design of near-allpass strictly stable minimal-phase real-valued rational iir filters. IEEE Trans. Circ. Syst. II: Trans. Briefs, 55: 781-785. DOI: 10.1109/TCSII.2008.922417

Jovanovic-Dolecek, G. and J. Diaz-Carmona, 2002. One structure for efficient narrow-band bandpass FIR filters. Proceedings of the 45th IEEE International Midwest Symposium, Aug. 4-7, IEEE Xplore Press, 485-488. DOI: 10.1109/MWSCAS.2002.1187079

Kulkarni, P.N. and P.C. Pandey, 2008. Optimizing the comb filters for spectral splitting of speech to reduce the effect of spectral masking. Proceedings of the IEEE International Conference on Signal Processing, Communications and Networking, Jan. 4-6, IEEE Xplore Press, Chennai, pp: 69-73. DOI: 10.1109/ICSCN.2008.4447163

Kulkarni, P.N., P.C. Pandey and D.S. Jangamashetti, 2007. Multi-band frequency compression for reducing the effects of spectral masking. Int. J. Speech Technol., 10: 219-227. DOI: $10.1007 / \mathrm{s} 10772-009-9030-\mathrm{z}$ 
Lian, Y. and Y. Wei, 2005. A computationally efficient nonuniform FIR digital filter bank for hearing aids. IEEE Trans. Circ. Syst. Regular Works, 52: 2754-2762. DOI: 10.1109/TCSI.2005.857871

Lim, Y., 1986. Frequency-response masking approach for the synthesis of sharp linear phase digital filters. IEEE Trans. Circ. Syst., 33: 357-364. DOI: 10.1109/TCS.1986.1085930

Lim, Y.C., Y.J. Yu, K.L. Teo and T. Saramaki, 2007. FRM-based FIR filters with optimum finite wordlength performance. IEEE Trans. Signal Proc., 55: 2914-2924. DOI: 10.1109/TSP.2007.893965

Lunner, T., S. Arlinger and J. Heligren, 1993. Channel digital filter bank for hearing aid use: Preliminary results in monaural, diotic and dichotic models. Scand. Audiol. J., 36: 75-81. PMID: 8153568

Lyregaard, P.E., 1982. Frequency selectivity and speech intelligibility in noise. Scand. Audiol. J.,15: 113-122. PMID: 6955919

Boer, E.D. and J. Bouwmeester, 1974. Critical bands and sensorineural hearing loss. Scandinavian Audiol. J., 13: 236-259.

Matei, R., 2007. Gaussian shaped multiband FIR and IIR 2D filters. Proceedings of the 14th IEEE International Conference on Electronics, Circuits and Systems, ICECS, Dec. 11-14, IEEE Xplore Press, Marrakech, pp: 254-257. DOI: 10.1109/ICECS.2007.4510978

Mintzer, F. and B. Liu, 1979. Practical design rules for optimum FIR bandpass digital filters. IEEE Trans. ASSP, 27: 204-206. DOI: 10.1109/TASSP.1979.1163205

Moore, B.C.J., 2003. An Introduction to the Psychology of Hearing. 5th Edn., Elsevier Academic Press, Amsterdam, ISBN-10: 0125056281 pp: 413.

Neuvo, Y., G. Rajan and S. Mitra, 1987. Design of narrow-band FIR bandpass digital filters with reduced arithmetic complexity. IEEE Trans. Circ. Syst., 4: 409-419. DOI: 10.1109/TCS.1987.1086149

Neuvo, Y., D. Cheng-Yu and S.K. Mitra, 1984. Interpolated finite impulse response filters. Proceedings of the IEEE Transaction Acoust Speech Signal Processing, ASSP, 32: 563-570. DOI: $10.1109 /$ TASSP.1984.1164348

Pelloni, D. and R. Lagadec, 1984. Enhancement of audio signals based on digital techniques. Proceedings of the IEEE International Conference on ICASSP Acoustics, Speech and Signal Processing, Mar. 19-21, IEEE Xplore Press, pp: 511-514. DOI: 10.1109/ICASSP.1984.1172455
Rabiner, L., J. Kaiser and R. Schafer, 1974. Some considerations in the design of multiband finiteimpulse-response digital filters. IEEE Transactions on Acoustics, Speech and Signal Processing, 22: 462-472. DOI: 10.1109/TASSP.1974.1162607

Rodrigues, J. and K.R. Pai, 2005a. Modified linear phase Frequency Response Masking FIR filter. Proceedings of the IEEE 4th International Symposium on Image and Signal Processing and Analysis, Sept. 15-17, IEEE Xplore Press, pp: 434439. DOI: 10.1109/ISPA.2005.195451

Rodrigues, J. and K.R. Pai, 2005b. New approach to the synthesis of sharp transition FIR digital filter. Proceedings of the IEEE Int. Symposium on Industrial Electronics, June 20-23, IEEE Xplore Press, $\quad$ pp: $1171-1173 . \quad$ DOI: 10.1109/ISIE.2005.1529090

Rodrigues, J., K.R. Pai and L.J. Gudino, 2006. Synthesis of linear phase sharp transition FIR digital filter. ECTI Trans. Electr. Eng., Electr. Commun., pp: 96-99.

Samadi, S., A. Nishihara, M.O. Ahmad and M.N.S. Swamy, 2004. Dedicated structure for tunable multiband FIR filters with linear phase and maximally flat magnitude. Proceedings of the IEEE Asia-Pacific Conference on Circuits and Systems, Dec. 6-9, IEEE Xplore Press, Tainan, pp: 413-416. DOI: 10.1109/APCCAS.2004.1412783

Selesnick, I.W., M. Lang and C.S. Burrus, 1998. A modified algorithm for constrained least square design of multiband FIR filters without specified transition bands. IEEE Trans. Signal Proc., 46: 497-501. DOI: 10.1109/78.655433

Shpak, D.J. and A. Antoniou, 1988. Two robust Remez methods for the design of FIR digital filters meeting prescribed specifications. Proceedings of the IEEE International Symposium on Circuits and Systems, Jun 7-9, IEEE Xplore Press, Finland, pp: 47-51. DOI: 10.1109/ISCAS.1988.14865

Yang, C.Z. and Y. Lian, 2002. Efficient prefilter structures for narrow-band bandpass FIR filter design. Proceedings of the IEEE Conference on Computers, Communication, Control and Power Electronics, Oct. 28-31, IEEE Xplore Press, pp: 893-896. DOI: 10.1109/TENCON.2002.1180264

Zahradnik, P. and M. Vleek, 2005. Analytical design method for optimal equiripple comb FIR filter. IEEE Circ. Syst. Briefs, 52: 112-115. DOI: 10.1109/TCSII.2001.840117

Zwicker, E., 1961. Subdivision of audio frequency range into critical bands. J. Acoust. Soc. Am., 33: 248248. 